\title{
Coproducción y desigualdad: recolección y residuos en barrios populares de la Región Metropolitana de Buenos Aires
}

Patricio-Bruno Besana. Universidad Nacional de General Sarmiento (UNGs), Buenos Aires, Argentina.

Ricardo-A. Gutiérrez. Consejo Nacional de Investigaciones Científicas y Técnicas (CONICET), Buenos Aires, Argentina.

RESUMEN | Este trabajo discute la relación entre coproducción de servicios a nivel local y desigualdad. Al respecto, se pregunta: ¿Qué formas asume regularmente la provisión local de servicios allí donde la sociedad civil es pobre y el Estado débil? ¿Qué resultados produce a nivel regional? Para responder dichas preguntas se desarrollaron 97 entrevistas semiestructuradas a funcionarios locales y se analizaron datos provenientes de fuentes secundarias sobre recolección y disposición intermedia de residuos en barrios populares de los 41 distritos que componen la Región Metropolitana de Buenos Aires. Los datos obtenidos indican que una gran variedad de actores coproducen servicios de menor calidad y extensión que los que produce el Estado por sí solo o a través de empresas privadas; y que dichos servicios alcanzan distintos resultados a escala local, favoreciendo desigualdades en el acceso a servicios a nivel agregado o regional.

PALABRAS CLAVE | servicios urbanos, desigualdad social, áreas metropolitanas.

ABSTRACT | This paper discusses the relationship between co-production of services at the local level and inequality. In this regard, we question: What forms does the local provision of services regularly assume where civil society is poor and the State is weak? What results does it produce at the regional level? To answer these questions, we performed 97 semi-structured interviews with local officials and analyzed data on the collection and intermediate disposal of waste in slums of the 41 districts of the Metropolitan Region of Buenos Aires, from secondary sources. The data shows that a great variety of actors co-produce services of lower quality and extension compared to those produced by the State alone or through private companies. Additionally, these services achieve different results at the local level, favoring unequal access to services at the aggregate or regional level.

KEYWORDS | urban services, social inequality, metropolitan areas.

Recibido el 6 de noviembre de 2020, aprobado el 31 de diciembre de 2020.

E-mails: P. Besana, pbesana@campus.ungs.edu.ar|R. Gutiérrez, rgutierr@unsam.edu.ar 


\section{Introducción}

A mediados de la década de 1990, tras años de relativo olvido, el concepto de coproducción comenzó a recuperar vigor (Williams et al., 2015). Elaborado con el fin de dar cuenta de la participación de consumidores en la provisión de servicios a escala local, y en ocasiones promoverla frente a la gestión pública centralizada, la coproducción ha sido objeto de debates que se prolongan hasta hoy día (Ahlers et al., 2014; Joshi \& Moore, 2004; Ostrom, 1996; Parks et al., 1981; Watson, 2014). Uno de los debates que provocó más controversias refiere a los efectos de la coproducción en la provisión de servicios en barrios o localidades pobres (vg. Brandsen \& Pestoff, 2006). Mientras algunos trabajos consideran la coproducción como la mejor alternativa frente a las fallas del mercado y del Estado (Joshi \& Moore, 2004; Ostrom, 1996), otros - por el contrario- ven en ella una forma de delegar responsabilidades en quienes menos recursos tienen (Ahlers et al., 2014). El panorama se completa con una tercera posición que pondera la coproducción solo bajo determinadas formas, contextos o circunstancias (Mitlin, 2008).

Pese a las diferencias sobre sus efectos, la literatura generalmente acuerda en que la coproducción es un fenómeno ampliamente extendido. Como resultado del ímpetu de la literatura por elaborar una teoría formal, gran parte de los trabajos sobre coproducción se fundaron en aportes meramente teóricos (Osborne et al., 2016; Osborne \& Strokosch, 2014) y en estudios de uno o pocos casos locales (Bovaird, 2007; Brandsen \& Pestoff, 2006; Mitlin, 2008; Watson, 2014). En ese sentido, pese al consenso sobre la extensión de la coproducción, son raros los estudios basados en una gran cantidad de casos. Si bien estudios de uno o pocos casos suelen recomendarse para elaborar teoría formal (vg. Neiman \& Quaranta, 2006), tanto por la escala de análisis local que abordan como por la cantidad pequeña de casos que incluyen, resultan insuficientes para analizar uno de los problemas que se pretende abordar aquí: los efectos de la coproducción sobre la igualdad o desigualdad en el acceso a servicios a nivel agregado.

Frente a dicho vacío, este trabajo busca hacer un aporte a partir de la comparación entre distritos que conforman una región metropolitana donde la provisión de servicios está en manos de actores locales. Al respecto, este estudio se pregunta: ¿Qué formas asume regularmente la provisión local de servicios alli donde la sociedad civil es pobre y el Estado débil? ¿Qué resultados produce a nivel regional?

En respuesta a la primera pregunta, se argumenta que la provisión local de servicios asume regularmente una diversidad de formas en las que una gran variedad de actores (co)producen servicios de menor calidad y extensión que los que produce el Estado por sí solo o a través de empresas privadas. En respuesta a la segunda pregunta, se sostiene que dicha variedad de formas y actores que (co)producen servicios alcanzan distintos resultados a nivel local, favoreciendo desigualdades en el acceso a servicios a nivel agregado o regional.

En términos sustantivos, este estudio se centra en la provisión de servicios de recolección y disposición intermedia ${ }^{1}$ de residuos sólidos urbanos (de aquí en

1 Por tal, se entiende al almacenamiento transitorio de residuos domiciliarios o asimilables en tachos, contenedores, campanas, tolvas u otros equipamientos antes de ser recogidos y enviados a sitios de recuperación o de disposición final, según corresponda. 
adelante RSU) en barrios populares de los 41 distritos que componen la Región Metropolitana de Buenos Aires (de aquí en adelante RMBA). La RMBA es el conglomerado urbano más grande de la Argentina y el que concentra más de la mitad de las familias que residen en barrios populares del país. A diferencia de los barrios formales, los barrios populares se han originado a partir de un conjunto de estrategias de acceso a la tierra que presentan diversos grados de precariedad, déficit de acceso formal a servicios públicos y situación dominial irregular (тЕсHо, 2016).

La selección del universo de análisis fue intencional conforme a tres rasgos considerados teóricamente relevantes: la diversidad de jurisdicciones con distintos niveles de gobierno que componen la región, la debilidad generalizada de los Estados locales en el sector de los RSU y la pobreza de la población que reside en barrios populares. El primero de esos rasgos es relevante en tanto condición de posibilidad. Por esta se entiende la selección de casos o universos a partir de rasgos que indican que el resultado de interés para la investigación podría ocurrir (cf. Mahoney \& Goertz, 2006). En general, se considera que Estados federales y/o regiones con distintos niveles de gobierno tienen más posibilidades de presentar experiencias de coproducción (Ostrom, 1999). Por un lado, esto se atribuye a la cercanía entre Estado local y sociedad, lo que promovería con mayor facilidad espacios de cooperación. Por otro lado, se suponen tantas posibilidades de coproducción como cantidad de gobiernos e instituciones públicas hay en una región. El segundo de los elementos mencionados (debilidad de los Estados locales) es relevante en tanto la coproducción se propone como alternativa a la provisión pública allí donde el Estado presenta dificultades para hacerse cargo de ella (Parks et al., 1981). Este sería el caso de los gobiernos locales que deben disponer de gran parte de su presupuesto a la hora de proveer los servicios de RSU. Finalmente, el tercer elemento (la pobreza) es pertinente porque, en contextos de debilidad estatal, la coproducción sería una alternativa sobre todo para quienes cuentan con pocas posibilidades económicas para acudir al mercado a la hora de suplir la falta o deficiente provisión de servicios públicos (Ostrom, 1996). En su conjunto, los aspectos relevantes para la selección del universo (diversidad de jurisdicciones que componen la región, debilidad estatal y pobreza de la sociedad civil) son definidos teóricamente y delimitados metodológicamente con mayor profundidad en los siguientes apartados.

El diseño de investigación se basó en la recopilación de datos de una diversidad de fuentes secundarias sobre provisión de servicios y población residente en barrios populares, la elaboración de datos propios sobre presupuestos municipales y el análisis de información obtenida mediante 97 entrevistas semiestructuradas a funcionarios de todos los Estados locales de la RMBA. ${ }^{2}$ Consistentemente con el tipo de muestreo intencional, el número final de entrevistas analizadas siguió los lineamientos del principio de saturación teórica (Holton \& Glaser, 2012).

Sigue a continuación un apartado teórico dedicado a repasar los antecedentes de la literatura sobre coproducción, así como los aspectos originales de esta propuesta. 
Allí se definen, entre otras nociones, los conceptos de formas regulares y alternativas de provisión de servicios, coproducción, pobreza y debilidad estatal. En el apartado que le sigue, se describe la RMBA y se presentan datos empíricos relativos a los tres rasgos considerados teóricamente relevantes para la selección del universo de análisis. A partir del repaso de diversas fuentes, en dicho apartado se muestra el acceso desigual a servicios de RSU en barrios populares y formales de la RMBA. Posteriormente, se analiza la información proveniente de las entrevistas. Allí se muestra cómo los actores que participan de la provisión de servicios en barrios populares y las formas en que lo hacen se distinguen de las que tienen lugar en barrios formales y entre sí según el distrito al que pertenezcan. En esa línea, también se muestra cómo dichas diferencias se traducen en resultados desiguales entre barrios populares y formales y entre distritos. Finalmente, el artículo presenta una discusión de los resultados alcanzados y aporta una serie de reflexiones sobre la relación entre coproducción, pobreza, informalidad y desigualdad.

\section{Coproducción en perspectiva teórica}

Los primeros antecedentes sobre coproducción se remontan a las décadas de 1960 y 1970 y se relacionan con la búsqueda de alternativas locales que superen la provisión centralizada de servicios (Garn et al., 1976; Ostrom \& Whitaker, 1973, 1974; Percy, 1978). Sin embargo, la noción acaba de tomar forma a principios de 1980, cuando un grupo de investigadores no solo reconoce la coproducción como una alternativa frente al aumento de las presiones fiscales, sino que además sugiere que "no resultaría -inequívocamente- en la provisión de servicios de menor calidad que los brindados por el Estado" (Parks et al., 1981, p. 1002).

Tras perder terreno hacia mediados de los 1980, la noción de coproducción comienza a recuperarlo una década más tarde (eg. Ahlers et al., 2014; Bovaird, 2007; Brandsen \& Pestoff, 2006; Gutberlet, 2015; Joshi \& Moore, 2004; Mitlin, 2008; Osborne et al., 2016; Swyngedouw, 2004; Watson, 2014; Williams et al., 2015). Popularizada por Elinor Ostrom (1996) como "un proceso a través del cual los esfuerzos de individuos que no provienen de la misma organización son transformados en bienes y servicios" (p. 1073), se utilizó para describir un sinfín de prácticas y arreglos formales e informales.

Por un lado, algunos trabajos trataron de circunscribir el campo de análisis a las cooperaciones regulares entre agencias del Estado y grupos organizados de ciudadanos (Joshi \& Moore, 2004, p. 21). Por otro lado, algunos estudios trataron de ampliarlo más allá de la esfera pública (Bovaird, 2007). Independientemente de ello, numerosos trabajos construyeron una gran diversidad de tipos ideales. Diferentes enfoques distinguen modalidades de coproducción según el momento en que la sociedad civil participa de ella (Brandsen \& Pestoff, 2006); según el nivel individual o colectivo de dicha participación (Bovaird, 2007; Brandsen \& Pestoff, 2006); y según quien toma la iniciativa de la coproducción, sea la sociedad civil o el Estado (Mitlin, 2008; Watson, 2014).

Más recientemente, estudios provenientes de otra perspectiva postularon la inevitabilidad de la coproducción de servicios y ponderaron al empoderamiento 
ciudadano por encima, incluso, de los servicios coproducidos (vg. Gutberlet, 2015; Osborne et al., 2016; Osborne \& Strokosch, 2014; Otsuky, 2016; Williams et al., 2015). Finalmente, análisis centrados en casos del Sur Global propiciaron un giro conceptual (vg. Swyngedouw, 2004) que pasó de poner el acento en la cooperación entre productores regulares (Estado y mercado) y actores locales, a considerar la coproducción como resultado de la colaboración entre actores formales e informales (eg. Chi et al., 2006; Guha-Khasnabis \& Kanbur, 2006; Medina, 2007; MorenoSánchez \& Maldonado, 2006; Nzeadibe, 2009; Tokman, 2007; Van Ginneken, 1999; Wilson et al., 2006).

Adicionalmente, dicho giro conceptual también propició la emergencia de una tradición más crítica (Ahlers et al., 2014; deWit \& Berner, 2009; Swyngedouw, 2004). Mientras los primeros trabajos coinciden en que la coproducción es la mejor forma posible de provisión de servicios allí donde el Estado es débil y/o la sociedad es pobre (eg. Joshi \& Ayee, 2008; Joshi \& Moore, 2004; Ostrom, 1996), trabajos críticos la sitúan en un contexto de gobernanza más allá del Estado (Swyngedouw, 2004), en donde este delega responsabilidades que le son propias y se produce una transformación de la idea de ciudadanía, que pasa de la participación en la escena pública al consumo en la esfera privada (Walsh, 1995). Mientras los primeros estudios concuerdan en que la cooperación o coordinación entre actores es un elemento importante de la coproducción (vg. Guha-Khasnabis \& Kanbur, 2006), los segundos desconfían de las posibilidades de implementarla en favor de los actores más débiles (eg. Ahlers et al., 2014; deWit \& Berner, 2009; Mitlin, 2008; Swyngedouw, 2004; Watson, 2014).

Finalmente, la idea de informalidad presente en trabajos críticos también difiere de la de aquellos estudios que promueven la coproducción. Mientras unos trabajos la ven como opuesta y separada de la acción del Estado, otros la consideran como una esfera coproducida por el mismo, ya sea por acción u omisión. En ese sentido, si para algunos estudios la coproducción es un modo de fortalecer y liberar el potencial de sectores e instituciones informales (Ostrom, 1999), para otros es una forma de mantenerlos en la informalidad, deslindando responsabilidades a la hora de garantizar el goce pleno de derechos y el acceso a ciertos servicios (eg. Ahlers et al., 2014; deWit \& Berner, 2009; Swyngedouw, 2004).

Pese a las notorias diferencias entre las perspectivas revisadas, tanto desde un punto de vista teórico como respecto de los resultados alcanzados, metodológicamente la mayoría de los trabajos consisten en estudios de uno o pocos casos a nivel local. Esto trae aparejada una serie de limitaciones. En primer lugar, conlleva el riesgo de tomar por regulares elementos que no lo son o lo son para ciertos escenarios y servicios (vg. Joshi \& Moore, 2004). En segundo lugar, supone una dificultad a la hora de controlar variables (vg. Gutberlet, 2015). En tercer lugar, no aporta una mirada de los efectos que la coproducción genera a nivel agregado - es decir, más allá de la escala local-. Todo ello contribuye a que distintos trabajos y tradiciones alcancen resultados diametralmente opuestos.

En ese sentido, este estudio se centra en el análisis de servicios de recolección y disposición intermedia de RSU en barrios populares de los 41 distritos que componen la RMBA. Si bien los trabajos sobre coproducción en el sector de los RSU 
son relativamente comunes, se enfocan -salvo contadas excepciones (van Horen, 2004) - en la inclusión de recuperadores urbanos -o actores informales- en modelos de gestión formal de RSU. Esto último es sumamente común entre los estudios desarrollados a partir de la experiencia de países o regiones del Sur global, América Latina y, particularmente, Argentina (vg. Medina, 2007; Moreno-Sánchez \& Maldonado, 2006; Nzeadibe, 2009). Por el contrario, aquí se propone analizar cómo el Estado produce o coproduce servicios en contextos considerados informales, a menudo con actores que también lo son.

En primer lugar, aquí se argumenta que allí donde la sociedad civil es pobre y el Estado débil, la provisión local asume regularmente una diversidad de formas en las que una gran variedad de actores (co)producen servicios de menor calidad y extensión que los que produce el Estado por sí solo o a través de empresas privadas. A priori, se asume que dicha variedad de formas se distingue de las implementadas por productores regulares (agencias del Estado o empresas privadas: Parks et al., 1981; Walsh, 1995) en los barrios formales de la RMBA. En línea con la propia literatura, distinguimos entre las formas regulares y las formas alternativas de provisión del servicio. Estas se diferencian de las primeras en los medios y recursos que utilizan, así como en los actores que participan de la producción de los servicios. En ese marco, por coproducción se entiende aquellos casos en que la provisión de servicios incluye a otros actores además de los productores regulares.

La idea de pobreza de la sociedad civil se vincula con una noción multidimensional que considera aspectos socioeconómicos (bajos ingresos), de derechos (violación/respeto de derechos civiles y políticos) y territoriales (tales como las dificultades de acceso formal a bienes y servicios públicos y a la tenencia de la tierra) (vg. Mitlin, 2008). En general, en los barrios populares se observan todos los aspectos que caracterizan la noción multidimensional de pobreza (тесно, 2016). A su vez, los aspectos territoriales presentan un vínculo estructural con la pobreza, puesto que quienes carecen de acceso formal a servicios o a la tierra donde residen tienen dificultades para escapar de la misma, incluso en periodos de fuerte redistribución del ingreso (Mitlin, 2008).

Por Estado débil, a su vez, se entiende al que cuenta con escasos recursos y capacidades en áreas que le competen (Alonso, 2007). Esta definición contempla la posibilidad de que un Estado sea fuerte en algunas áreas y débil en otras. En ese sentido, los recursos económicos son fundamentales para analizar la fortaleza de un Estado en la provisión de servicios que requieren de cierta infraestructura urbana (Alonso, 2007). Asimismo, las capacidades estatales también pueden verse reforzadas por medio de actores que cuentan con los recursos de los que el Estado carece (Alonso, 2007). Como veremos más adelante, si bien una experiencia exitosa de coproducción en escenarios donde el Estado es débil y la sociedad es pobre es una posibilidad teórica -tal como indican algunos estudios (eg. Joshi \& Ayee, 2008; Joshi \& Moore, 2004; Ostrom, 1996)-, los resultados de este trabajo señalan lo contrario. Concretamente, los datos sugieren que en áreas y servicios como los que analizamos, los Estados son débiles por falta de capacidades para financiar equipamiento e infraestructura urbana, precisamente un recurso del que carecen los más pobres. 
En segundo lugar, aquí se argumenta que las formas y actores que (co)producen servicios en barrios populares alcanzan distintos resultados a escala local, favoreciendo desigualdades en el acceso a servicios a nivel agregado o regional. Esos resultados incluyen el acceso, la frecuencia y la calidad de los servicios, así como la presencia de basurales ilegales.

Puesto que el estudio se centra en los efectos de la coproducción sobre la igualdad o desigualdad, los servicios prestados en barrios populares se evalúan en relación con aquellos logrados en barrios formales de cada distrito, para luego considerar diferencias entre barrios populares de los distintos distritos de la región. Al respecto, los resultados alcanzados muestran que la coproducción es un fenómeno relativamente extendido (tal como indica el conjunto de la literatura), al tiempo que presenta características variables tanto entre barrios populares y formales, como entre barrios del mismo tipo pero de distintos distritos. En este punto, el énfasis en lo local no parece hacerle un favor a la literatura, no solo en relación con la escala de análisis, sino también respecto del énfasis en el rol de los actores locales en la coproducción. Al respecto, este trabajo aporta datos relativos a la diversidad de actores que producen servicios y a los distintos resultados que alcanzan.

A continuación, se describe la RMBA a partir de las características que llevaron a considerarla como universo de análisis. Entre otras cuestiones, en el siguiente apartado se muestran datos que dan cuenta de algunas de las diferencias en materia de acceso a servicios de RSU en barrios formales y populares.

\section{RMBA: jurisdicciones múltiples, debilidad estatal y RSU en barrios populares}

La RMBA es el área más densamente poblada de la Argentina, con un 37\% de la población (cerca de 15 millones de personas) en menos del 1\% del territorio nacional (Instituto Nacional de Estadística y Censos [INDEC], 2010). Esta, tal como se mencionó en apartados anteriores, reúne una serie de rasgos que la hacen teóricamente relevante para el problema planteado: i) la cantidad y diversidad de jurisdicciones que la componen; ii) la debilidad de los Estados locales para brindar servicios de RSU; y iii) la situación de los barrios populares.

La RMBA se compone de 40 partidos de la Provincia de Buenos Aires y de la Ciudad Autónoma de Buenos Aires (de aquí en adelante CABA), un distrito federal con rango de provincia. Por ser distrito federal, la CABA goza de la autonomía que no tienen los anteriores, dependientes del Estado provincial. La CABA también cuenta con un presupuesto muy superior y presenta indicadores socioeconómicos más elevados que la gran mayoría de los partidos bonaerenses (Morales, 2017).

Si bien la RMBA no cuenta con una instancia de gestión metropolitana propiamente dicha, sí son varios los entes u organizaciones nacionales e interjurisdiccionales encargados de brindar o regular distintos servicios a lo largo de toda o parte de la región. En el sector de los RsU, se encuentra la Coordinación Ecológica Área Metropolitana Sociedad del Estado (CEAmse), dependiente de la CABA y de la Provincia en partes iguales. La CEAMSE es responsable de la disposición final de RSU, aunque no de la recolección de los mismos. 
Dicho esquema se relaciona con el segundo rasgo teóricamente relevante de la RMBA: la debilidad de los gobiernos locales a la hora de brindar servicios de RSU. Puesto que CEAmse solo es responsable por la disposición final de los residuos, la responsabilidad por su recolección y disposición transitoria recae en el gobierno de la CABA y de los partidos bonaerenses de la RMBA. Al costo de dichos servicios, se agrega el importe que percibe la CEAMSE por la disposición final.

TABLA I Gasto municipal en RSU en valores absolutos y en relación al gasto total para el año 2012

\begin{tabular}{|c|c|c|}
\hline DISTRITO & $\begin{array}{l}\text { GASTO EN RESIDUOS SÓLIDOS } \\
\text { URBANOS }\end{array}$ & $\%$ \\
\hline General San Martín & 166.975 .000 & 23 \\
\hline Tres de Febrero & 99.127 .000 & 20 \\
\hline Hurlingham & 40.781 .000 & 20 \\
\hline Morón & 113.831 .000 & 18 \\
\hline Escobar & 57.586 .000 & 17 \\
\hline Ituzaingó & 48.591 .000 & 16 \\
\hline Almirante Brown & 109.350 .000 & 16 \\
\hline San Isidro & 143.043 .000 & 16 \\
\hline San Fernando & 40.493 .000 & 15 \\
\hline La Plata & 161.251 .000 & 15 \\
\hline Pilar & 82.646 .000 & 15 \\
\hline Avellaneda & 146.791 .000 & 14 \\
\hline Tigre & 136.730 .000 & 14 \\
\hline Vicente López & 103.949 .000 & 14 \\
\hline San Miguel & 59.242 .000 & 14 \\
\hline Campana & 26.373 .000 & 11 \\
\hline Quilmes & 89.533 .000 & 11 \\
\hline Florencio Varela & 55.936 .000 & 11 \\
\hline Brandsen & 5.612 .000 & 11 \\
\hline Esteban Echeverría & 43.266 .000 & 10 \\
\hline General Rodríguez & 11.323 .000 & 9 \\
\hline Berisso & 16.758 .000 & 9 \\
\hline Ensenada & 16.574 .000 & 9 \\
\hline Presidente Perón & 10.776 .000 & 9 \\
\hline Zárate & 15.689 .000 & 8 \\
\hline CABA & 2.842 .037 .889 & 8 \\
\hline Merlo & 63.277 .000 & 8 \\
\hline Luján & 13.880 .000 & 7 \\
\hline José C. Paz & 30.527 .000 & 6 \\
\hline General Las Heras & 2.042 .000 & 5 \\
\hline Berazategui & 26.201 .000 & 5 \\
\hline Exaltación de la Cruz & 4.721 .000 & 5 \\
\hline Cańuelas & 6.214 .000 & 3 \\
\hline Marcos Paz & 1.776 .000 & 1 \\
\hline Total & 4.878 .996 .889 & 10 \\
\hline Total sin caba & 2.036 .959 .000 & 12 \\
\hline
\end{tabular}

FUENTE: ELABORACIÓN PROPIA CON BASE EN DATOS DE LA DIRECCIÓN GENERAL DE CONTADURÍA DE LA CABA (2OI2) Y DIRECCIÓN TÉCNICA MUNICIPAL DE LA PROVINCIA DE BUENOS AIRES (2OI 2) 
En todos los casos, ya sea que los resuelvan por sus propios medios o a través de empresas privadas, los servicios de RSU representan una erogación importante para los gobiernos locales. Sin embargo, la CABA dispone para tales fines de recursos muy superiores a los de los partidos bonaerenses de la RMBA, incluso cuando algunos de estos asignen para ello mayores porcentajes de sus presupuestos. La Tabla 1 muestra el gasto en RSU para el año 2012 en valores absolutos y porcentuales para 34 distritos, incluyendo la CABA.

En el marco de una economía caracterizada por ostentar altas tasas de inflación anual y por recaudaciones que no se actualizan al tiempo que lo hacen los costos de los servicios (Schejtman \& Irurita, 2012), los partidos bonaerenses de la RMBA se encuentran -año a año- en una situación de creciente debilidad. A ello se suma el hecho de que suelen ser castigados electoralmente cuando los servicios de RSU no colman las expectativas de la mayoría de los contribuyentes (Montera, 2019). Es decir, descuidarlos no es una opción. Así lo muestra la Tabla 2, que incluye datos de 2010 sobre segmentos de hogares alcanzados por el servicio de recolección de $\mathrm{RSU}^{3}$ en 129 de los 135 partidos bonaerenses (Dirección de Estadística de la Provincia de Buenos Aires, 2011).

TABLA 2 Porcentaje de hogares alcanzados por el servicio de RSU de la Provincia de Buenos Aires

\begin{tabular}{|c|c|c|c|c|}
\hline OBSERVACIONES & MEDIA & $\begin{array}{c}\text { DESVÍO } \\
\text { ESTÁNDAR }\end{array}$ & MÍNIMO & MÁxIMO \\
\hline 129 & 96,6 & 2,4 & 86,5 & 99,5 \\
\hline
\end{tabular}

FUENTE: ELABORACIÓN PROPIA CON BASE EN DATOS DE LA DIRECCIÓN DE ESTADÍSTICA DE LA PROVINCIA DE BUENOS AIRES (2OII)

Dichos porcentajes, no obstante, se reducen significativamente cuando se analiza la situación de quienes residen en barrios populares, la cual ilustra el tercer rasgo referido más arriba: la pobreza de los barrios populares en materia de acceso formal a servicios. Al respecto, la Tabla 3 compara datos sobre ausencia o acceso irregular a servicios públicos en barrios populares de los partidos bonaerenses de la RMBA en los periodos 2010-2011 y 2015-2016.

En general, se observa una retracción auspiciosa de la ausencia de servicios entre los períodos analizados. En particular, la falta de recolección frecuente de residuos ${ }^{4}$ es la que registra una mayor retracción intermuestral $(10,9)$. No obstante, al tiempo que se reduce la falta de recolección frecuente, la tabla muestra un incremento en la presencia de basurales ilegales en las inmediaciones de los barrios populares. ${ }^{5}$

3 Refiere a la existencia en el segmento de servicio regular de recolección de residuos (al menos dos veces por semana).

4 Refiere a una frecuencia de recolección de, al menos, día por medio.

5 Refiere al porcentaje de hogares con presencia de basurales en las inmediaciones de sus viviendas. La fuente no brinda precisiones sobre lo que entiende por "inmediaciones". 
TABLA 3 | Ausencia o acceso irregular a servicios en barrios populares de partidos bonaerenses de RMBA

\begin{tabular}{|l|c|c|c|}
\hline \multicolumn{1}{|c|}{ SERVICIO } & 20I0-20I I & 20I 5-20I6 & VARIACIÓN \\
\hline Agua & 42,3 & 33,1 & $(-9,2)$ \\
\hline Energía eléctrica & - & 29,7 & $(-3,1)$ \\
\hline Gas natural & 88,9 & 85,8 & $(-4,3)$ \\
\hline Cloacas & 83,3 & 79,0 & $(-1,8)$ \\
\hline Desagües pluviales & 73,9 & 72 & $(-10,9)$ \\
\hline Recolección de Rsu frecuente & 28,5 & 17,6 & $(5,2)$ \\
\hline Presencia de basurales & 50,2 & 55,4 & \\
\hline
\end{tabular}

FUENTE: ELABORACIÓN PROPIA CON BASE EN BONOFIGLIO (2OI7)

Algo similar indican datos referidos a barrios populares de la CABA (тесно, 2016). Allí el porcentaje de acceso $(85,4 \%$ ) y la frecuencia del servicio de recolección (entre 5 y 6 veces a la semana) es mayor al que muestran barrios populares de otros nueve territorios, incluyendo la Provincia de Buenos Aires (теснo, 2016). No obstante, pese a que los residuos generados en la CABA deben disponerse en su totalidad en el relleno sanitario Norte in de la CEAMSE, la misma fuente indica que 70,8\% de los generados en barrios populares serían dispuestos en basurales a cielo abierto. Es decir, tanto datos referidos a la Provincia de Buenos Aires, como a la CABA, señalan que un alto porcentaje de los residuos generados en barrios populares no se dispone donde debería.

Los datos sobre déficits en la recolección y disposición transitoria de RSU o presencia de basurales no son menores si se considera la cantidad de barrios populares que se localizan en la RмBa. Para el año 2018, la caba y la Provincia de Buenos Aires concentraban la mayor cantidad de familias residiendo en ellos, con un $61 \%$ entre CABA $(10,5 \%)$ y Provincia de Buenos Aires (50,5\%) (Registro Nacional de Barrios Populares [RENABAP], 2020). Por su parte, los distritos bonaerenses de la RMBA concentraban el $92,84 \%$ de las familias (unas 390.340) que residen en barrios populares de la provincia, siendo que ninguno de esos distritos carece de dichos barrios (Registro Público Provincial de Villas y Asentamientos Precarios [RPpVAP], 2019).

En suma, hasta aquí se mostró cómo la RMBA se compone de distintas jurisdicciones y gobiernos locales, cuán costoso es para la mayoría de los últimos prestar servicios de RSU y cómo los barrios populares, que concentran una cantidad considerable de familias, serían los más perjudicados a la hora de acceder a dichos servicios.

La literatura sobre capacidades estatales, así como parte de la literatura sobre coproducción, indica que Estados con recursos insuficientes a menudo pueden fortalecer sus capacidades a partir de la colaboración con otros actores que aporten esos u otros recursos. En el apartado que sigue se presentan los resultados de las entrevistas a funcionarios locales, con objeto de dar cuenta de las formas y características que asumen los servicios de RSU en barrios populares. Puntualmente, se pone a prueba si la coproducción de servicios de RSU atenúa o no la debilidad estatal en materia de recursos económicos. 


\section{Coproducción y servicios de RSU en barrios populares}

Con objeto de cotejar las formas y resultados de la provisión de servicios de RSU en barrios populares en relación con aquella de los barrios formales de los 41 distritos de la RMBA, se realizaron 97 entrevistas semiestructuradas a funcionarios locales. Se entrevistó, al menos, a dos funcionarios competentes por distrito (en general, pertenecientes a las carteras de Obras y Servicios Públicos y/o de Ambiente) en carácter de unidades de recolección de información, siendo los distritos las unidades de análisis.

Los resultados obtenidos arrojaron que, en 30 distritos (73\%), los servicios de RSU en barrios populares se llevarían a cabo por vías alternativas a las desarrolladas en barrios formales; en tres distritos (7\%) no habría diferencias, y respecto de los ocho restantes $(20 \%)$ no se dispuso de información certera. En estos últimos, los funcionarios entrevistados otorgaron información contradictoria, no quisieron contestar o seńalaron no tener barrios populares en sus territorios. De aquí en adelante, este tipo de respuestas son categorizadas como Ns/Nc (Figura 1).

\section{FIGURA I | Servicios de RSU en barrios populares en relación con barrios formales}

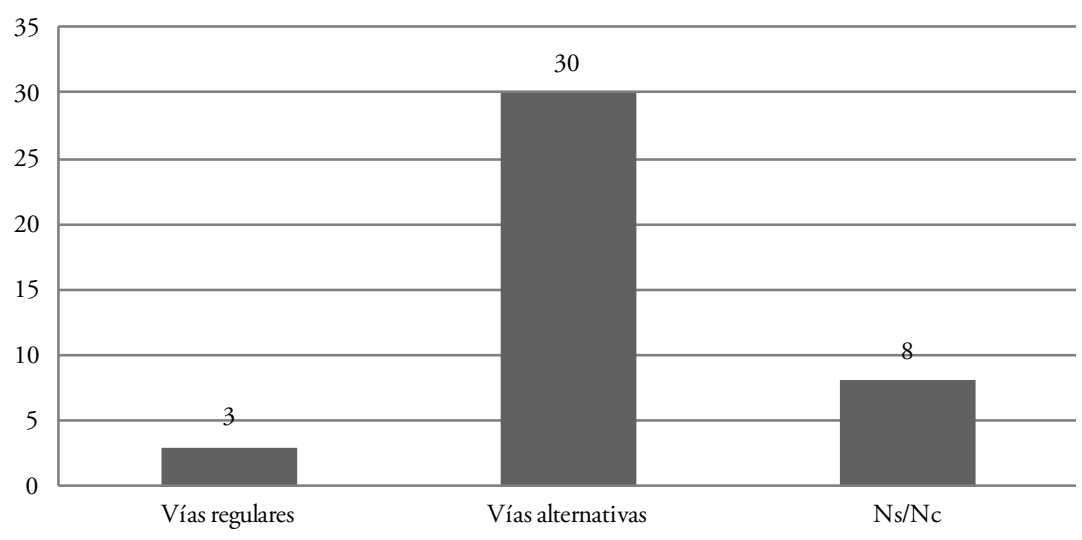

FUENTE: ELABORACIÓN PROPIA

Con excepción de quienes se negaron a contestar, las respuestas categorizadas como $\mathrm{Ns} / \mathrm{Nc}$ son inconsistentes con las de otros entrevistados del mismo distrito y/o con la información elaborada por las fuentes consignadas en el apartado anterior (TECHO, 2016; RPPVAP, 2019). Tanto por la negativa a contestar como por dichas inconsistencias, es muy probable que exista un subregistro respecto de las formas alternativas de servicios de RSU.

De aquí en más, los datos refieren a los 30 distritos que otorgaron información consistente respecto a formas alternativas de provisión de servicios en barrios populares. Un análisis del contenido de dicha información indica que las diferencias respecto de los barrios formales puede desagregarse según tres elementos: i) productores regulares y coproductores; ii) medios y equipamiento; y iii) frecuencia de la recolección. 


\section{Productores regulares}

Mientras los productores regulares refieren a las carteras públicas o a las empresas privadas que llevan a cabo los servicios en los barrios formales de cada distrito, los coproductores refieren a los actores sociales que colaboran en la prestación. En ese sentido, se encontraron diferencias en 21 casos respecto de las tareas que desarrollan los productores regulares en barrios populares y en barrios formales. Esos casos incluyen a todos los distritos que concesionan los servicios de RSU a empresas privadas (13). En ellos, los Estados locales deben hacerse cargo de prestar parte de los servicios en barrios populares, pese a haber concesionado el conjunto de los mismos en barrios formales a empresas privadas. En muchos casos, esto se da mediante la creación ad hoc de distintas carteras municipales. Casi por unanimidad (12), la razón señalada es la reticencia de las empresas privadas a llevar a cabo parte del servicio, generalmente la recolección, al interior de los barrios populares.

Si bien existen diferencias entre los servicios que prestan los productores regulares en barrios populares o en formales, la mayoría de los entrevistados los consideran como los principales productores de los servicios de RSU en ambos tipos de barrios. En ese sentido, en doce casos los productores principales son direcciones municipales (57\%); en cinco casos son empresas privadas (24\%); en tres, delegaciones municipales (14\%) y en uno, organizaciones de la sociedad civil (5\%).

Con independencia de quién es identificado como el productor principal, 21 distritos contarían con actores sociales como coproductores, en ocho no se obtuvo información concluyente y tan solo uno no contaría con actores sociales en el rol mencionado (Figura 2).

\section{FIGURA 2 | Coproductores en barrios populares}

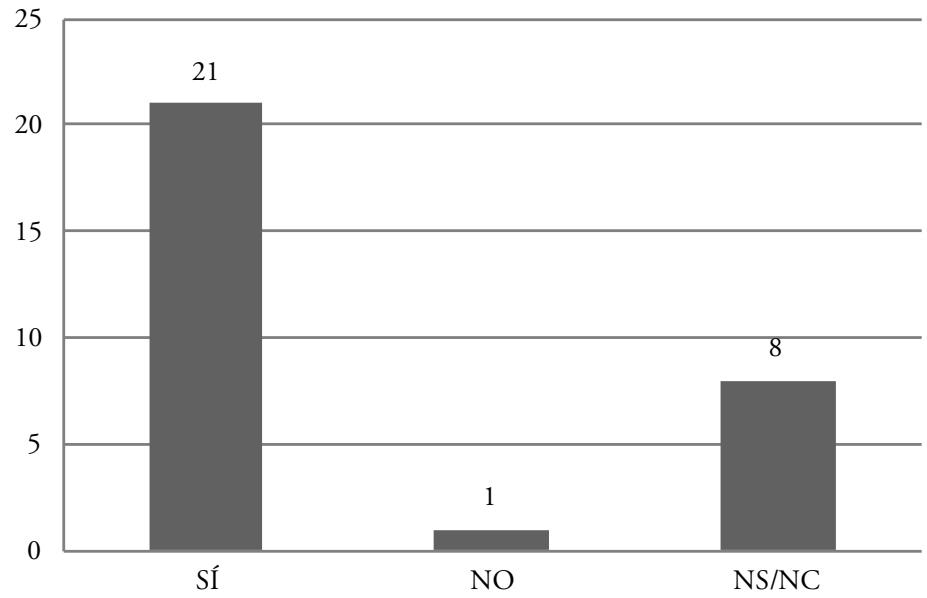

FUENTE: ELABORACIÓN PROPIA 
Los tipos de actores sociales se distribuyen del siguiente modo: once distritos contarían con cooperativas de trabajo auspiciadas por el Estado, ${ }^{6}$ nueve con cooperativas autónomas, ${ }^{7}$ siete con carreros $^{8}$ y cuatro con brokers o punteros politicos. Vale señalar que, en ocasiones, más de un tipo de actor tiene presencia en barrios populares de un mismo distrito, razón por la cual el total de actores supera el total de los 21 distritos.

En el caso de las cooperativas autónomas y promovidas por el Estado (20 en total), se observa algún tipo de arreglo, contrato o cuasi contrato que da cierta formalidad a la cooperación Estado-sociedad; eso no ocurre entre los punteros o brokers y entre los carreros (11 en total). Estos últimos, en general, parecen desarrollar una actividad individual sin ninguna o con muy poca colaboración del Estado.

Con excepción de un caso, no se obtuvo información que indique que la coproducción de servicios es iniciativa de productores regulares o de actores sociales. Más bien, los relatos refieren a "esfuerzos independientes" (sobre todo en el caso de los carreros), demandas atendidas "como se puede" o "con los recursos que se tienen a disposición", o a "servicios solidarios a quienes no abonan tasas".

\section{Medios y equipamiento}

En lo que respecta a la categoría medios, referida al transporte o locomoción con que se lleva a cabo la recolección, también se observaron medios alternativos en 21 distritos, no se observaron en uno de ellos y en ocho no se obtuvo información concluyente. Así puede observarse en la Figura 3.

\section{FIGURA 3 | Medios de recolección alternativos}

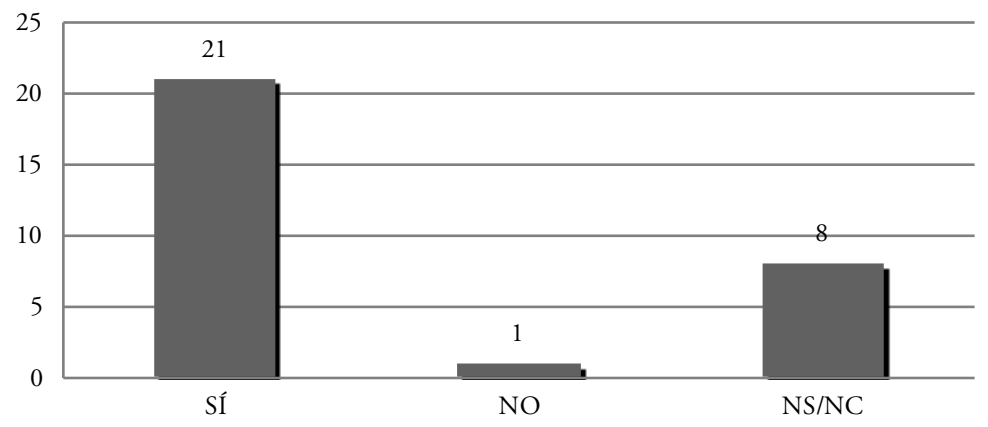

FUENTE: ELABORACIÓN PROPIA

$6 \longdiv { \text { Refieren a agrupaciones de vecinos promovidas por el Estado para el desarrollo de obras y servicios } }$ a cambio del financiamiento que otorgan distintos programas de transferencia condicionada.

7 Refieren a cooperativas que no habrían sido creadas por el Estado, ni dependen exclusivamente de su financiamiento.

8 Denominación que usualmente se les da a personas de a pie o a caballo que tiran de carros y recogen los residuos en zonas en las que no pueden ingresar vehículos pesados o de gran porte. 
Los 21 casos que utilizan medios de transporte alternativos se distribuyen del siguiente modo: en seis la recolección se lleva a cabo por medio de carros a tracción a sangre, ya sea tirado por equinos o por sus propios dueńos; en otros seis, la recolección la realizan personas de a pie, sin carro o transporte alguno; en tres, los entrevistados no supieron o no quisieron contestar; en otros tres, la recolección involucra distintos medios, según las características particulares de cada barrio popular; en dos casos, la recolección se lleva a cabo por medio de tractores; y en un caso, se emplea un camión de pequeńas dimensiones.

Respecto del equipamiento utilizado para la disposición transitoria de los RSU, se observan diferencias en 26 distritos. No obstante, la distribución es menos variada que la de los medios de recolección. Una categoría agrupa al 69\% de los casos relevados: el uso de tolvas o volquetes en barrios populares, a diferencia de las campanas o contenedores comúnmente utilizados en barrios formales. Los volquetes son cajones abiertos, generalmente utilizados para residuos áridos o voluminosos y con una capacidad de carga que suele variar de 5 a 17 metros cúbicos. Los contenedores son dispositivos cerrados, con bocas laterales o superiores, específicamente elaborados para la disposición de RSU y con una capacidad de carga significativamente menor. Mientras que al uso de contenedores o campanas se lo ha denominado contenerización, denominamos al de volquetes como volqueterización. Por lo general, la volqueterización consiste en la disposición de un volquete por barrio popular en algún lugar que facilite el acceso de camiones volqueteros para ser retirados. En suma, la Figura 4 indica cómo, de los 26 distritos que emplean equipamientos alternativos en barrios populares, 18 apelan a la volqueterización.

\section{FIGURA 4 | Equipamiento para disposición transitoria de RSU}

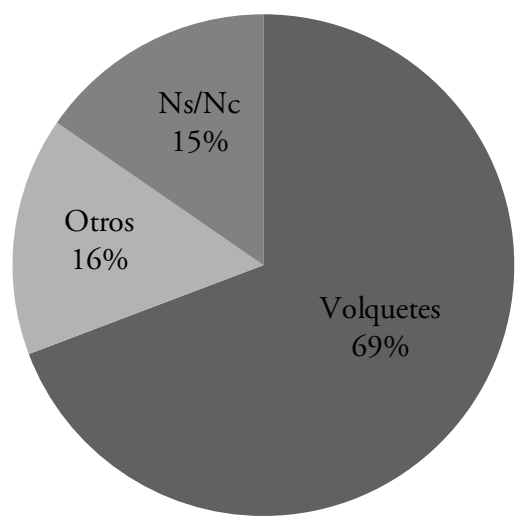

FUENTE: ELABORACIÓN PROPIA

$\mathrm{Al}$ respecto, es llamativo que los mismos funcionarios que utilizan los volquetes reconocen que su gran capacidad de carga hace que no sean vaciados o reemplazados con regularidad, facilitando la generación de microbasurales. 


\section{Frecuencia de la recolección}

Finalmente, la frecuencia de recolección, referida a la cantidad de veces semanales en que se recogen los residuos, varía entre tres y seis días en barrios formales, según el distrito. Teniendo dicha frecuencia como referencia, en solo cinco distritos no se registrarían diferencias entre esos barrios y los barrios populares. Los restantes distritos se distribuyen del siguiente modo: en quince la frecuencia en barrios populares sería menor, en ocho no se obtuvo información concluyente, en uno sería mayor en barrios populares, y en el restante se afirmó que no sería menor, pero no se brindaron seguridades sobre si es mayor o igual que en barrios formales. Así lo muestra la Figura 5.

FIGURA 5 | Frecuencia de recolección en barrios populares en relación con barrios formales

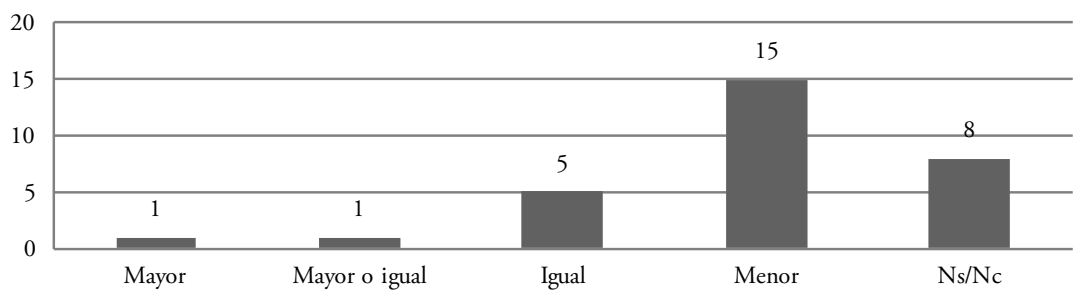

FUENTE: ELABORACIÓN PROPIA

En los casos en que la frecuencia es menor, las razones esgrimidas se relacionan con déficits de infraestructura, agravados en días de lluvia: el mal estado de los caminos, la falta de asfalto para que circulen medios de transporte apropiados, las dimensiones angostas de los pasillos, entre otras. Asimismo, en diez de los quince distritos donde la frecuencia sería menor, la recolección se llevaría a cabo entre dos y cuatro veces a la semana; en dos casos, tres veces; en otros dos, entre tres y una vez; y en el restante la frecuencia sería irregular.

En resumen, la información presentada señala diferencias entre los servicios prestados en barrios populares o en barrios formales de la RMBA. Adicionalmente, la información obtenida también muestra diferencias entre los distintos distritos en lo que respecta a los productores y coproductores, los medios de recolección y equipamientos de disposición intermedia y la frecuencia con que se llevan a cabo los servicios en los barrios populares. Toda esta información agrega elementos no observados hasta el momento por la literatura sobre coproducción. En torno a ello, se organiza la discusión que sigue.

\section{Discusión}

La impresión de que la coproducción es más común de lo que usualmente se cree parece ser el único gran consenso sobre el tema tras cuarenta años de discusiones. No obstante, la adopción de un enfoque local por parte de la mayoría de los trabajos 
sobre coproducción a lo largo de todo el periodo, no aportó suficientes datos empíricos para constatar dicha impresión. En ese marco, este trabajo adoptó una mirada regional o agregada de escenarios locales para poner a prueba el mencionado consenso. La información obtenida al respecto confirma que servicios de RSU en barrios populares de la RMBA, esto es, en escenarios locales de pobreza y debilidad estatal, suelen desarrollarse por vías alternativas (30 casos sobre 41, 70\%) y que, dentro de ellas, predomina la coproducción (21 casos). Asimismo, la adopción de una mirada regional también permitió observar diferencias entre distritos de una misma región, algo que un enfoque a escala local no podría más que presumir.

En ese sentido, este estudio dio cuenta, en primer lugar, de la gran variedad de actores sociales que coproducen servicios en distintos escenarios. En segundo lugar, se pudo observar que una parte considerable de los coproductores (20) coopera con los Estados locales mediante algún tipo de arreglo institucional (Joshi \& Moore, 2004), aunque un segmento considerable (11) no lo hace formalmente (Ahlers et al., 2014). En tercer lugar, el estudio mostró cómo productores regulares (15 carteras públicas, entre direcciones y delegaciones municipales, y 5 empresas privadas) continúan siendo los principales productores de servicios en barrios populares y cómo, entre estos, las carteras públicas prestarían servicios allí donde las empresas privadas se niegan a hacerlo. En cuarto lugar, el trabajo mostró que, aunque productores regulares tienen un rol protagónico en la coproducción, los medios y equipamientos que se destinan a servicios de RSU en barrios populares tienden a diferir de los utilizados en barrios formales, siendo más precarios y pudiendo estar vinculados a problemas como la presencia de basurales. En quinto lugar, se pudo observar que en el 50\% de los distritos (15) donde se proveen servicios de forma alternativa, en barrios populares se prestan con menor frecuencia que en barrios formales. Esto se observa en un contexto general donde -ya de por sí- la frecuencia con que se proveen servicios por vías regulares en barrios formales también varía según el distrito (generalmente, entre tres y seis días semanales).

En resumen, tanto las formas que asume la coproducción (productores regulares, coproductores, medios y equipamiento) como los resultados que generan (alta presencia de basurales y baja frecuencia de recolección) dan cuenta de servicios de menor calidad que los provistos por productores regulares en barrios formales. Estos resultados son coherentes con los menores porcentajes de provisión de servicios presentados previamente (RPPVAP, 2019; TECHO, 2016). Los datos relevados también indican diferencias en los resultados alcanzados por los distritos de la región, ya sea en barrios formales o en barrios populares. En conjunto, los datos indican que las desigualdades en el acceso a los servicios no parecen atenuarse mediante la coproducción y otras formas alternativas de provisión de servicios. Sobre este y otros temas se discute en el siguiente y último apartado.

\section{Conclusiones}

Quien haya leído recientemente estudios sobre administración pública o provisión de servicios probablemente esté habituado al término coproducción. No obstante, su uso recurrente no parece ir acompañado de una mayor acumulación de 
conocimiento. Como ya se señaló, la adopción de un enfoque local por parte de la mayoría de los trabajos no favoreció la generación de consensos. Basta mirar cómo, mientras algunos estudios ilustran experiencias exitosas de coproducción, otros similares llaman la atención sobre sus pésimos resultados. Adicionalmente, la proliferación de casi tantos tipos ideales como estudios sobre coproducción y el estiramiento conceptual que ello trajo aparejado generaron mayores confusiones que certezas (vg. Mitlin, 2008). Es decir, de modo similar a lo que Sartori (2002) sugirió para la idea moderna de política, la palabra coproducción "está en boca de todos, pero ya no sabemos pensar la cosa" (p. 216).

En ese contexto de incertidumbre, este trabajo se centró en la discusión sobre coproducción allí donde la sociedad es pobre y el Estado débil. A preguntas como por qué la coproducción habría de ser exitosa en contextos de pobreza, con qué recursos cuentan los pobres para fortalecer o compensar las capacidades de Estados débiles, algunos trabajos responden que los más pobres cuentan con mayor tiempo libre e instituciones informales adaptadas a sus espacios vitales (Meagher, 2013; Ostrom, 1996). Por instituciones informales se refieren a un acervo de conocimientos, prácticas, formas de organizaciones (comunitarias o relacionadas al caciquismo o clientelismo) que cumplirían un rol en la provisión de servicios. Efectivamente, muchos de los actores locales identificados (carreros, punteros, entre otros) se relacionan con dicho acervo y desempeñan una función en la coproducción de servicios. No obstante, este trabajo mostró que la coproducción en barrios populares alcanza peores resultados que los alcanzados por productores regulares en barrios formales. En otras palabras, si bien es una posibilidad teórica que una agregación o sinergia virtuosa de actores y recursos resulte en una experiencia exitosa de coproducción -tal como indican algunos de los estudios citados-, no parece ser la norma en escenarios donde el Estado es débil y la sociedad es pobre, que paradójicamente es donde más suele recomendarse.

Más bien, en áreas y servicios como los que analizamos, los Estados son débiles por la falta de capacidades para financiar equipamiento e infraestructura urbana, precisamente un recurso del que carecen los más pobres. En ese sentido, recordemos que la pobreza es un fenómeno multidimensional que incluye, además de ingresos insuficientes, la falta de acceso formal a servicios públicos. También recordemos que, en el caso de la pobreza estructural, la falta de servicios es la que, en gran medida, dificulta que sectores de la población eleven su calidad de vida incluso en contextos de redistribución del ingreso. En suma, parece extraordinariamente difícil lograr que sectores populares accedan a servicios de los que carecen y que requieren de sumas de dinero de las que no disponen, delegando en ellos parte de las responsabilidades por dichos servicios.

Ahora bien, además de la relación entre coproducción y escenarios de pobreza, este estudio se preguntó por el vínculo entre coproducción e igualdad. Como fuera seńalado, los resultados mostraron que la coproducción es un fenómeno relativamente extendido, al tiempo que presenta características variables a nivel regional. En este punto, nuevamente el énfasis de la literatura sobre coproducción en la escala local no parece hacerle un favor. No obstante, aquí el problema no solo refiere a la escala local de análisis, sino también al énfasis en el rol de los actores locales en la 
coproducción. Siendo locales, ¿por qué no habrían de variar los actores (sociales, estatales y privados) que forman parte de la coproducción o las formas que adquiere esta última en cada caso? ¿Por qué no habrían de hacerlo los resultados que alcanzan? Esto último quizá no sea un problema en el marco de servicios que requieren de menor financiamiento público o respecto de sectores de mejores ingresos que los aquí analizados; es decir, en sociedades donde cierto nivel de bienestar e igualdad social está garantizado. Ciertamente, en esos contextos varios trabajos vinculan la coproducción con cierto empoderamiento ciudadano (Meagher, 2013; Osborne et al., 2016). No obstante, allí donde esto no ocurre, apelar a la coproducción para reducir la desigualdad en el acceso a servicios, más que fomentar el derecho a la libre elección o a la participación de sectores vulnerables, parece consolidar las condiciones de informalidad y desigualdad que pesan sobre ellos.

En vistas de inconvenientes como los mencionados, trabajos afines a la coproducción señalan que esta no es la mejor alternativa, sino la mejor posible (Joshi \& Ayee, 2008; Joshi \& Moore, 2004), considerando -implícita o explícitamente- la coproducción como una solución paliativa. Ahora bien, si los Estados son débiles a la hora de prestar ciertos servicios, ¿no vale más fortalecerlos que transferir responsabilidades? ¿Por qué la coproducción es la primera o única respuesta? ¿Será acaso que las instituciones informales que algunos trabajos gustan ensalzar son el resultado de la retirada del Estado más que un refuerzo de sus capacidades? Por último, si la provisión de servicios en manos de actores locales (incluyendo a los Estados) resulta en un escenario desigual, más que delegar responsabilidades, ¿no convendría recorrer el camino inverso y centralizar los servicios? ¿Acaso no sería mejor que la participación de actores locales se limitara al control de servicios prestados por productores regulares? Las anteriores son preguntas para las cuales no es fácil encontrar respuestas satisfactorias. Por lo pronto, tal como se observó en este trabajo, deslindando responsabilidades en quienes menos tienen, los Estados parecen coproducir lo que dicen querer combatir: pobreza, precariedad, informalidad y desigualdad en el acceso a servicios.

\section{Agradecimientos}

Los autores agradecen a Máximo Lanzetta, a los miembros del Área de Ambiente y Política (AAP) de la Escuela de Política y Gobierno (epyg) de la Universidad Nacional de San Martín (UnSAM) y a los evaluadores anónimos convocados por EURE, por sus comentarios y sugerencias.

\section{Referencias bibliográficas}

Ahlers, R., Cleaver, F., Rusca, M. \& Schwartz, K. (2014). Informal space in the urban waterscape: Disaggregation and coproduction of water services. Water Alternatives, $7(1), \quad 1-14 . \quad$ https://www.water-alternatives.org/index.php/alldoc/articles/vol7/ v7issue1/230-a7-1-1/file

Alonso, G. V. (Ed.). (2007). Capacidades estatales, instituciones y politica social. Prometeo. 
Bonofiglio, N. (2017). Informe Técnico. Acceso a condiciones habitacionales, infraestructura urbana básica y a un medio ambiente saludable para la población del Conurbano Bonaerense. Observatorio de la Deuda Social Argentina y Defensoría de la Provincia de Buenos Aires. https://www.defensorba.org.ar/pdfs/informes-tecnicos-upload-2019/informetecnico-medioambiente.pdf

Bovaird, T. (2007). Beyond engagement and participation: User and community coproduction of public services. PAR - Public Administration Review, 67(5), 846-860. https://doi. org/10.1111/j.1540-6210.2007.00773.x

Brandsen, T. \& Pestoff, V. (2006). Coproduction, the third sector and the delivery of public services. Public Management Review, 8(4), 493-501. https://doi. org/10.1080/14719030601022874

Chi, X., Streicher-Porte, M., Wang, M. Y. L. \& Reuter, M. A. (2006). Informal electronic waste recycling: A sector review with special focus on China. Waste Management, 31(4), 731774. https://doi.org/10.1016/j.wasman.2010.11.006

deWit, J. \& Berner, E. (2009). Progressive patronage? Municipalities, Ngos, своs and the limits to slum dwellers' empowerment. Development and Change, 40(5), 925-947. https://doi.org/10.1111/j.1467-7660.2009.01589.x

Dirección de Estadística de la Provincia de Buenos Aires. (2011). Datos de Hogares por segmento por servicio 2010. Provincia de Buenos Aires. [Documento de Trabajo]. Dirección de Estadística de la Provincia de Buenos Aires.

Dirección General de Contaduría, Ciudad Autónoma de Buenos Aires (CABA). (2012). Informe de Balance Anual, 2012. Gobierno de la Ciudad Autónoma de Buenos Aires.

Dirección Técnica Municipal de la Provincia de Buenos Aires. (2012). Informe técnico municipal, tercer cuatrimestre 2012. Subsecretaría de Asuntos Municipales, Gobierno de la Provincia de Buenos Aires.

Garn, H., Flax, M., Springer, M. \& Taylor, J. (1976). Models for indicator development: A framework for policy analysis. The Urban Institute.

Guha-Khasnabis, B. \& Kanbur, R. (2006). Micro-insurance for the informal economic workers in India. En B. Guha-Khasnabis, R. Kanbur \& E. Ostrom, Linking the formal and informal economy. UNU-WIDER Studies in Development Economics (pp. 165-189). Oxford University Press.

Gutberlet, J. (2015). More inclusive and cleaner cities with waste management coproduction: Insights from participatory epistemologies and methods. Habitat International, 46, 234-243. https://doi.org/10.1016/j.habitatint.2014.10.004

Holton, J. \& Glaser, B. (2012). The grounded theory review methodology reader: Selected papers 2004-2011. Sociology Press.

Instituto Nacional de Estadística y Censos (INDEC), Argentina. (2010). Censo Nacional 2010. INDEC. https://www.indec.gob.ar/indec/web/Nivel4-Tema-2-41-135

Joshi, A. \& Ayee, J. (2008). Associational taxation: A pathway into the informal sector? Cambridge University Press.

Joshi, A. \& Moore, M. (2004). Institutionalised co-production: Unorthodox public service delivery in challenging environments. Journal of Development Studies, 40(4), 31-49. https://doi.org/10.1080/00220380410001673184 
Mahoney, J. \& Goertz, G. (2006). The possibility principle: Choosing negative cases in comparative research. The American Politics Science Review, 98(4), 653-669. https:// doi.org/10.1017/S0003055404041401

Meagher, K. (2013). Unlocking the informal economy: A literature review on linkages between formal and informal economies in developing countries. Working Paper $\mathrm{N}^{\circ} 27$, Women in Informal Employment: Globalizing and Organizing (wIEGO). http://www.vumelana. org.za/wp-content/uploads/2014/03/Document-49-Meagher_WIEGO_WP27.pdf

Medina, M. (2007). The world's scavengers: Salvaging for sustainable consumption and production. Rowman Altamira.

Mitlin, D. (2008). With and beyond the state: co-production as a route to political influence, power and transformation for grassroots organizations. Environment and Urbanization, 20, 339-360. https://doi.org/10.1177/0956247808096117

Montera, C. (2019). De la transparencia a la integralidad: La construcción de la agenda de gestión de residuos en el municipio de Morón (1999-2015). XIV Congreso Nacional de Ciencia Politica "La politica en incertidumbre. Reordenamientos globales, realineamientos domésticos y la cuestión de la transparencia", Universidad Nacional de San Martín, Buenos Aires.

Morales, M. (2017). ¿Cómo gasta el AMBA? Ejecución presupuestaria de los gobiernos de CABA y el Conurbano - 2016. Serie Documentos Cem N ${ }^{\circ}$ 7. Centro de Estudios Metropolitanos (CEM). http://estudiosmetropolitanos.com.ar/2017/08/29/como-gasta-el-amba/

Moreno-Sánchez, R. del P. \& Maldonado, J. (2006). Surviving from garbage: The role of informal waste-pickers in a dynamic model of solid-waste management in developing countries. Environment and Development Economics, 11(3), 371-391. https://doi. org/10.1017/S1355770X06002853

Neiman, G. \& Quaranta, G. (2006). Los estudios de caso en la investigación sociológica. En I. Vasilachis de Gialdino (Ed.), Estrategias de investigación cualitativa (pp. 213-238). Gedisa.

Nzeadibe, T. C. (2009). Solid waste reforms and informal recycling in Enugu urban area, Nigeria. Habitat International, 33(1), 93-99. https://doi.org/10.1016/j.habitatint.2008.05.006

Osborne, S., Radnor, Z. \& Strokosch, K. (2016). Co-production and the co-creation of value in public services: A suitable case for treatment? Public Management Review, 18(5), 639-653. https://doi.org/10.1080/14719037.2015.1111927

Osborne, S. \& Strokosch, K. (2014). It takes two to tango? Understanding the coproduction of public services by integrating the services management and public administration perspectives. British Journal of Management, 24, 31-47. https://doi.org/10.1111/14678551.12010

Ostrom, E. (1996). Crossing the great divide: Coproduction, synergy, and development. World Development, 24(6), 1073-1087. https://doi.org/10.1016/0305-750X(96)00023-X

Ostrom, E. (1999). Principios de diseño y amenazas a las organizaciones sustentables que administran recursos comunes. De cara a la globalización, organizaciones económicas de América Latina y el Caribe [Conferencia]. http://www.fidamerica.cl/actividades/ conferencias/oec/ostroesp.html

Ostrom, E. \& Whitaker, G. (1973). Does local community control of police make a difference? Some preliminary findings. Midwest Political Science Association Stable, 17(1), 48-76. https://doi.org/10.2307/2110474 
Ostrom, E. \& Whitaker, G. (1974). Community control and governmental responsiveness: The case of police in Black neighborhoods (Microficha $N^{\circ} 15411$; Improving the Quality of Urban Management, pp. 304-334). National Criminal Justice Reference Service. https://www.ojp.gov/pdffiles1/Digitization/15411NCJRS.pdf

Otsuky, K. (2016). Infrastructure in informal settlements: Coproduction of public services for inclusive governance. Local Environment. The International Journal of Justice and Sustainability, 2(12), 1557-1572. https://doi.org/10.1080/13549839.2016.1149456

Parks, R. B., Baker, P. C., Kiser, L., Oakerson, R., Ostrom, E., Ostrom, V., Percy, S. L., Vandivort, M. B., Whitaker, G. \& Wilson, R. (1981). Consumers as coproducers of public services: Some economic and institutional considerations. Indiana University and The University of North Carolina.

Percy, S. L. (1978). Conceptualizing and measuring citizen coproduction of safety and security. Policy Studies Journal, 7, 486-492.

Registro Nacional de Barrios Populares (RenABAP), Argentina. (2020, febrero 14). Argentina. gob.ar. https://www.argentina.gob.ar/desarrollosocial/renabap

Registro Público Provincial de Villas y Asentamientos Precarios (RPpVAP), Argentina. (2019). http://190.188.234.6/registro/publico/

Sartori, G. (2002). La política: Lógica y método en las ciencias sociales. Fondo de Cultura Económica.

Schejtman, L. \& Irurita, N. (2012, diciembre). Diagnóstico sobre la gestión de residuos sólidos urbanos en municipios de la Argentina. Documentos de Trabajo CIPPEC [Centro de Implementación de Políticas Públicas para la Equidad y el Crecimiento], № 103. https://www.cippec.org/wp-content/uploads/2017/03/1552.pdf

Swyngedouw, E. (2004). Social power and the urbanization of water: Flows of power. Oxford University Press.

теCHо. (2016). Relevamiento de Asentamientos Informales 2016. теСHо Argentina. http://datos. techo.org/dataset/argentina-relevamiento-asentamientos-informales-2016

Tokman, V. (2007). The informal economy, insecurity and social cohesion in Latin America. International Labour Review, 146(1-2), 82-107. https://doi.org/10.1111/j.1564913X.2007.00006.x

Van Ginneken, W. (Ed.). (1999). Social security for the excluded majority: Case studies of developing countries. International Labour Organization (ILO).

van Horen, B. (2004). Fragmented coherence: Solid waste management in Colombo. International Journal of Urban and Regional Research, 28(4), 757-773. https://doi. org/10.1111/j.0309-1317.2004.00550.x

Walsh, K. (1995). Public services and market mechanisms: Competition, contracting and the New Public Management. Palgrave Macmillan.

Watson, V. (2014). Co-production and collaboration in planning: The difference. Planning Theory \& Practice, 15(1), 62-67. https://doi.org/10.1080/14649357.2013.866266

Williams, B. N., Kang, S.-C. \& Johnson, J. (2015). (Co)-contamination as the dark side of co-production: Public value failures in co-production processes. Public Management Review, 18(5), 692-717. https://doi.org/10.1080/14719037.2015.1111660

Wilson, D. C., Velis, C. \& Cheeseman, C. (2006). Role of informal sector recycling in waste management in developing countries. Habitat International, 30(4), 797-808. https:// doi.org/10.1016/j.habitatint.2005.09.005 